\title{
Magnetic helicity and eruptivity in active region 12673
}

\author{
K. Moraitis ${ }^{1}$, X. Sun ${ }^{2}$, É. Pariat ${ }^{1}$, and L. Linan ${ }^{1}$ \\ ${ }^{1}$ LESIA, Observatoire de Paris, Université PSL, CNRS, Sorbonne Université, Université de Paris, 5 place Jules Janssen, \\ 92195 Meudon, France \\ e-mail: kmorait@phys.uoa.gr \\ 2 Institute for Astronomy, University of Hawaii at Manoa, Pukalani, HI 96768-8288, USA
}

Received 11 May 2019 / Accepted 13 July 2019

\begin{abstract}
Context. In September 2017, the largest X-class flare of solar cycle 24 occurred from the most active region (AR) of this cycle, AR 12673. This AR attracted much interest because of its unique morphological and evolution characteristics. Among the parameters that were examined in the AR was magnetic helicity, but either only approximately, or intermittently, or both.

Aims. We here study the evolution of the relative magnetic helicity and of the two components of its decomposition, the non-potential, and the volume-threading one, in the time interval around the highest activity of AR 12673 . We especially focus on the ratio of the non-potential to total helicity, which has recently been proposed as an indicator of AR eruptivity.

Methods. We first approximated the coronal magnetic field of the AR with two different optimization-based extrapolation procedures, and chose the method that produced the most reliable helicity value at each instant. Moreover, in one of these methods, we weighted the optimization by the uncertainty estimates derived from the Helioseismic and Magnetic Imager (HMI) instrument for the first time. We then followed an accurate method to compute all quantities of interest.

Results. The first observational determination of the evolution of the non-potential to total helicity ratio seems to confirm the quality it has in indicating eruptivity. This ratio increased before the major flares of AR 12673 and afterwards relaxed to lower values. Additionally, we discuss the evolution patterns of the various helicity and energy budgets of AR 12673 and compare them with results from other works.
\end{abstract}

Key words. Sun: activity - magnetohydrodynamics (MHD) - Sun: flares - methods: numerical

\section{Introduction}

Magnetic helicity is a physical quantity that is used often in investigations of the eruptive behaviour of solar active regions (ARs, Rust 1994; Nindos \& Andrews 2004; Green et al. 2018) because it is a conserved quantity of ideal magnetohydrodynamics (MHD), and thus it determines the dynamics of magnetized systems (Taylor 1974; Pariat et al. 2015; Linan et al. 2018). Magnetic helicity is a geometrical quantity that describes the level of complexity of a magnetic field, through the twist and writhe of its field lines, and their interlinking.

Magnetic helicity, however, is well defined only for systems that are magnetically closed, and thus is of limited use in the Sun and all other astrophysical conditions. The appropriate form of helicity in these situations is relative magnetic helicity (Berger \& Field 1984; Finn \& Antonsen 1985). It is defined through a volume integral as

$H=\int_{\mathrm{V}}\left(\boldsymbol{A}+\boldsymbol{A}_{\mathrm{p}}\right) \cdot\left(\boldsymbol{B}-\boldsymbol{B}_{\mathrm{p}}\right) \mathrm{d} V$

where $\boldsymbol{B}$ denotes the 3D magnetic field in the volume of interest, $V$, and $\boldsymbol{B}_{\mathrm{p}}$ is a reference field that usually is taken to be potential. The two fields are generated from the vector potentials $\boldsymbol{A}$ and $\boldsymbol{A}_{\mathrm{p}}$. The potential magnetic field is chosen to have the same normal components with $\boldsymbol{B}$ along the boundary of the volume, $\partial V$,

$\left.\hat{n} \cdot \boldsymbol{B}\right|_{\partial V}=\left.\hat{n} \cdot \boldsymbol{B}_{\mathrm{p}}\right|_{\partial V}$

with $\hat{n}$ denoting the unit vector that is normal to $\partial V$. This choice guarantees that the relative magnetic helicity given by Eq. (1) is independent from the gauges of the vector potentials, and thus is physically meaningful.

Relative magnetic helicity can also be uniquely split into two gauge-independent components (Berger 1999),

$H=H_{\mathrm{j}}+H_{\mathrm{pj}}$.

These are the non-potential component

$H_{\mathrm{j}}=\int_{\mathrm{V}}\left(\boldsymbol{A}-\boldsymbol{A}_{\mathrm{p}}\right) \cdot\left(\boldsymbol{B}-\boldsymbol{B}_{\mathrm{p}}\right) \mathrm{d} V$

which depends only on the current-carrying part of the magnetic field, $\boldsymbol{B}_{\mathrm{j}}=\boldsymbol{B}-\boldsymbol{B}_{\mathrm{p}}$, and the volume-threading component

$H_{\mathrm{pj}}=2 \int_{\mathrm{V}} \boldsymbol{A}_{\mathrm{p}} \cdot\left(\boldsymbol{B}-\boldsymbol{B}_{\mathrm{p}}\right) \mathrm{d} V$

which additionally depends on the potential field. The detailed analysis of the behaviour of these components and of their dynamics in Linan et al. (2018) showed that unlike relative magnetic helicity, they are not conserved quantities of ideal MHD.

The study of the different components of relative magnetic helicity is important, however, as they provide additional information compared to $H$. Already from the first separate examination of these two components in eruptive ARs (Moraitis et al. 2014), it was observed that the non-potential component fluctuates in accordance with the eruptions of the AR. This was noted in both observed and synthetic MHD-modelled eruptive ARs.

Moreover, using this decomposition of helicity in a set of eruptive and non-eruptive MHD flux-emergence simulations, 
Pariat et al. (2017) have determined that the ratio $\left|H_{\mathrm{j}}\right| /|H|$ is an excellent indicator of the system eruptivity. They noted that the helicity ratio behaved differently in the eruptive than in the non-eruptive simulations, as well as between the pre- and posteruptive phases of the former. The helicity ratio obtained high values only in the eruptive cases, and only before the eruptions. The possible importance of $\left|H_{\mathrm{j}}\right| /|H|$ was also highlighted in Linan et al. (2018) using an MHD simulation of the formation of a coronal jet. Again, the helicity ratio attained very high values during the generation of the jet, and it dropped significantly after that.

This result was further tested in Zuccarello et al. (2018) with a different set of line-tied eruptive MHD simulations where the quality of the helicity ratio as an eruptivity indicator was reconfirmed. Additionally, by carefully identifying the onset of the eruption in these simulations, it was found that a threshold value of $\left|H_{\mathrm{j}}\right| /|H| \simeq 0.3$ was reached by all simulations when the eruptions occurred.

These promising results on the ratio $\left|H_{\mathrm{j}}\right| /|H|$ led to an interest in deriving it in observations. In a first observational determination of the helicity ratio, James et al. (2018) have found the value $\left|H_{\mathrm{j}}\right| /|H| \simeq 0.17$ for the flux rope of an AR one hour before the occurrence of an eruption. A reasonable next step is to study the evolution of the helicity ratio in an observed AR, which is the central task of this work.

A perfect target for this is the most active region of Solar Cycle 24, AR 12673 (Sun \& Norton 2017). During its passage across the solar disc in the week of 4-10 September 2017, it produced four X-class flares, including the two strongest of the cycle, $27 \mathrm{M}$-class flares, and numerous more of smaller size, as the Geostationary Operational Environmental Satellite (GOES) soft X-ray (0.1-0.8 nm) light curve of Fig. 1 depicts.

A few of these flares also produced coronal mass ejections (CMEs). Of the three halo CMEs, two were Earth-directed and geoeffective (Chertok et al. 2018), while one of them led to particle acceleration at the produced shock front (Morosan et al. 2019), and also to a ground-level enhancement of energetic particles (Augusto et al. 2019). This particle event originated from an X8.2 flare and its associated CME of 10 September 2017 (Veronig et al. 2018), when AR 12673 was crossing the west solar limb.

Most of the interest in the activity of AR 12673 was focused on the two X-class flares of 6 September 2017, however; the confined X2.2 flare that started at 08:57 UT (SOL2017-0906T08:57), and the eruptive X9.3 flare, the largest since 2005, that followed three hours after the first, at 11:53 UT (SOL201709-06T11:53). In this work, we are interested in a ten-hour time interval centred around the X2.2 flare of 6 September that also includes the X9.3 flare.

The photospheric morphology of AR 12673 started as a single positive-polarity sunspot and quickly became quite complicated, displaying a complex network of $\delta$-sunspots. It exhibited significant flux emergence and the highest instantaneous flux rate ever observed (Sun \& Norton 2017), with values reaching up to $10^{21} \mathrm{Mx} \mathrm{h}^{-1}$ on 3 September, as Fig. 1 also shows. Many dipoles emerged close to the initial sunspot, and the successive interactions between them resulted in the increased complexity of the system.

The magnetic field of the AR as measured by the Helioseismic and Magnetic Imager (HMI, Scherrer et al. 2012) instrument on board the SDO was very strong, especially along the polarity inversion line (Wang et al. 2018). Photospheric shearing and twisting motions were also observed (Verma 2018), which helped in the creation of the highly non-potential configuration that powered the two flares.
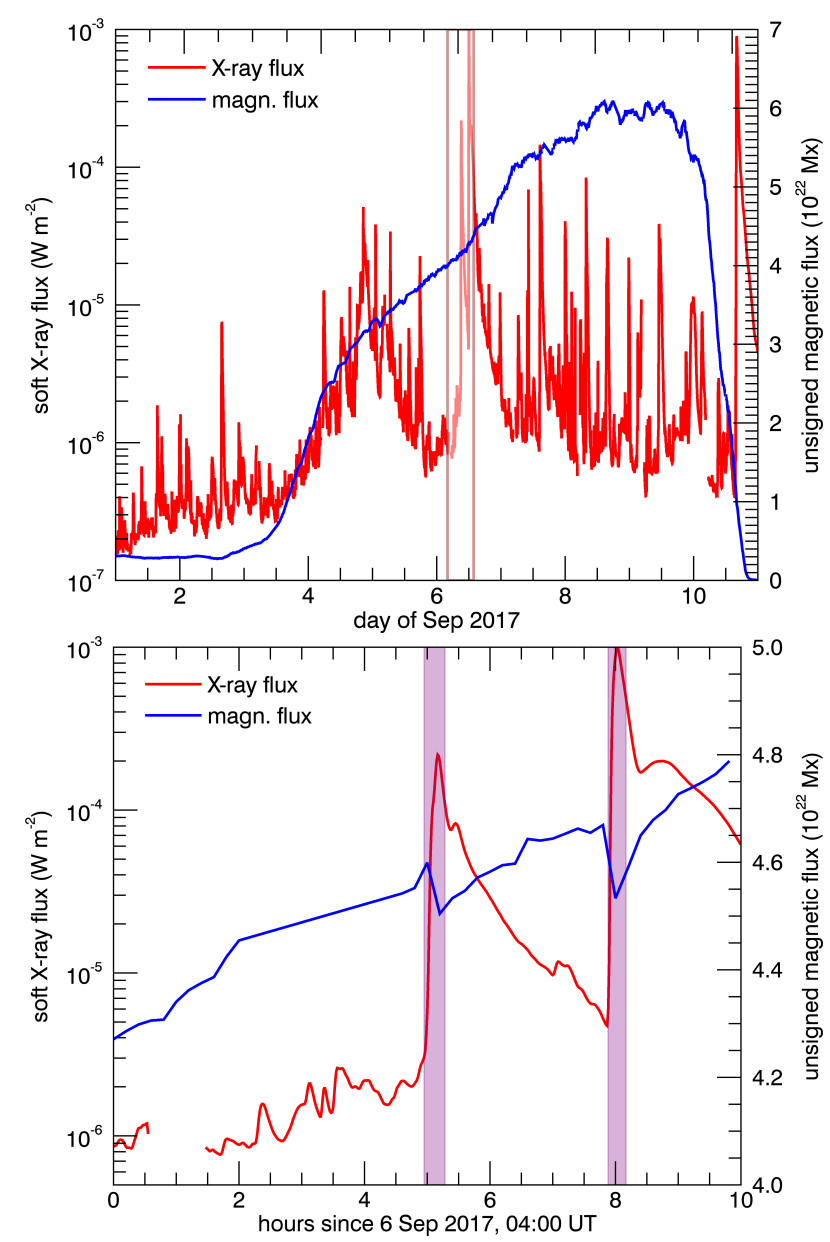

Fig. 1. GOES soft X-ray light curve and evolution of HMI unsigned magnetic flux in AR 12673 for the first ten days of September 2017 (top). The brown vertical lines denote the interval of interest for this study, which is shown zoomed in at the bottom. The purple bands in this plot denote the time intervals of the X2.2 and the X9.3 flares, with onset times at 08:57 UT and 11:53 UT, respectively. The HMI magnetic flux in the bottom plot is of limited accuracy due to the emission of the flares, while between 06:00 UT and 08:36 UT there were no observations due to an eclipse of the Solar Dynamics Observatory (SDO) (Pesnell et al. 2012).

Many models were invoked in order to explain the evolution of AR 12673 towards the two X-class flares and the eruption of the second. Yang et al. (2017) associated the increased flare-productivity of the AR with the blocking of newly emerging flux by already existing flux, and the eruptive flare with a filament becoming kink unstable. The formation of a coronal sigmoid and the initiation of both X-class flares from the core of the sigmoid led Mitra et al. (2018) to characterize the AR as a single "sigmoid-to-arcade" event.

Hou et al. (2018) attributed the generation of the two flares to the formation of multiple flux ropes and twisted loop bundles that eventually erupted. A large highly-twisted magnetic flux rope and some smaller flux ropes were also confirmed by the MHD modelling of the X9.3 flare of AR 12673 made by Inoue et al. (2018).

The highly twisted flux ropes that were found by many authors indicate that magnetic helicity probably is an important factor in the eruptivity of AR 12673. Liu et al. (2018) have found a significant enhancement, by almost a factor of three, of the magnetic helicity during the confined X2.2 flare, although they approximated helicity only with its twist. In another study, 
Yan et al. (2018) did not find a significant change in helicity during the first $\mathrm{X}$-class flare, but only a small decrease during the second. They found a negative helicity injection rate throughout the day of the two flares, however, which is consistent with the rotational motions of the sunspots in AR 12673.

The sign of magnetic helicity was negative for the whole week of the AR activity, as was deduced by integrating the helicity injection rate (Vemareddy 2019). Vemareddy (2019) also found that AR 12673 exhibited very fast helicity injection, by a factor of three higher than other ARs when it was normalized to the square of magnetic flux.

All these findings support the choice of AR 12673 as an interesting case to study the evolution of the helicity ratio in an observed AR. The question we address is whether this evolution is related with the eruptivity of the AR, as proposed by Pariat et al. (2017). In addition to $\left|H_{\mathrm{j}}\right| /|H|$, we are also interested in examining the evolution of all helicity- and energy-related budgets of the AR. To determine all quantities of interest, the coronal magnetic field must first be estimated. The method we followed for this is described in Sect. 2. The results that we obtained are presented in Sect. 3, and they are then discussed in Sect. 4.

\section{Method}

This section describes the method we followed to compute all magnetic helicities and energies in AR 12673. We first describe the non-linear force-free (NLFF) field extrapolation method that we used in order to approximate the coronal magnetic field of the AR. Then, we verified that the produced coronal fields were suitable for use with the helicity computation method, which we briefly describe afterwards.

\subsection{Coronal field modelling}

The starting point of all computations performed in this work were magnetic field data from the HMI instrument of SDO. We used the vector magnetogram data with 12 min cadence (product hmi.sharp_720s). The actual cadence of the data varied during the interval of interest due to an eclipse of the observatory and a few low-quality data during the two flares. The morphology of the $B_{z}$ distribution on the photosphere at the beginning and at the end of the ten-hour interval of study is shown in Fig. 2.

The next step was to model the 3D magnetic field of AR 12673 in a finite volume above the AR with an NLFF field extrapolation method. These methods try to simultaneously satisfy the force-free and the solenoidal conditions for the magnetic field, which read

$$
\begin{aligned}
& (\nabla \times \boldsymbol{B}) \times \boldsymbol{B}=0 \\
& \nabla \cdot \boldsymbol{B}=0 .
\end{aligned}
$$

The unavoidable presence of numerical errors in the solution process prevents the exact fullfilment of these conditions. The solenoidal condition is thus never fully constrained in an NLFF extrapolated magnetic field. The level of nonsolenoidality is very important for the helicity computations, however. Valori et al. (2016) have shown that the values of helicity are unreliable when the non-solenoidality errors are above a certain level. It is therefore essential to estimate the level of solenoidality of the given magnetic field when the relative magnetic helicity is computed, which we performed in two ways, as described in Sect. 2.2.
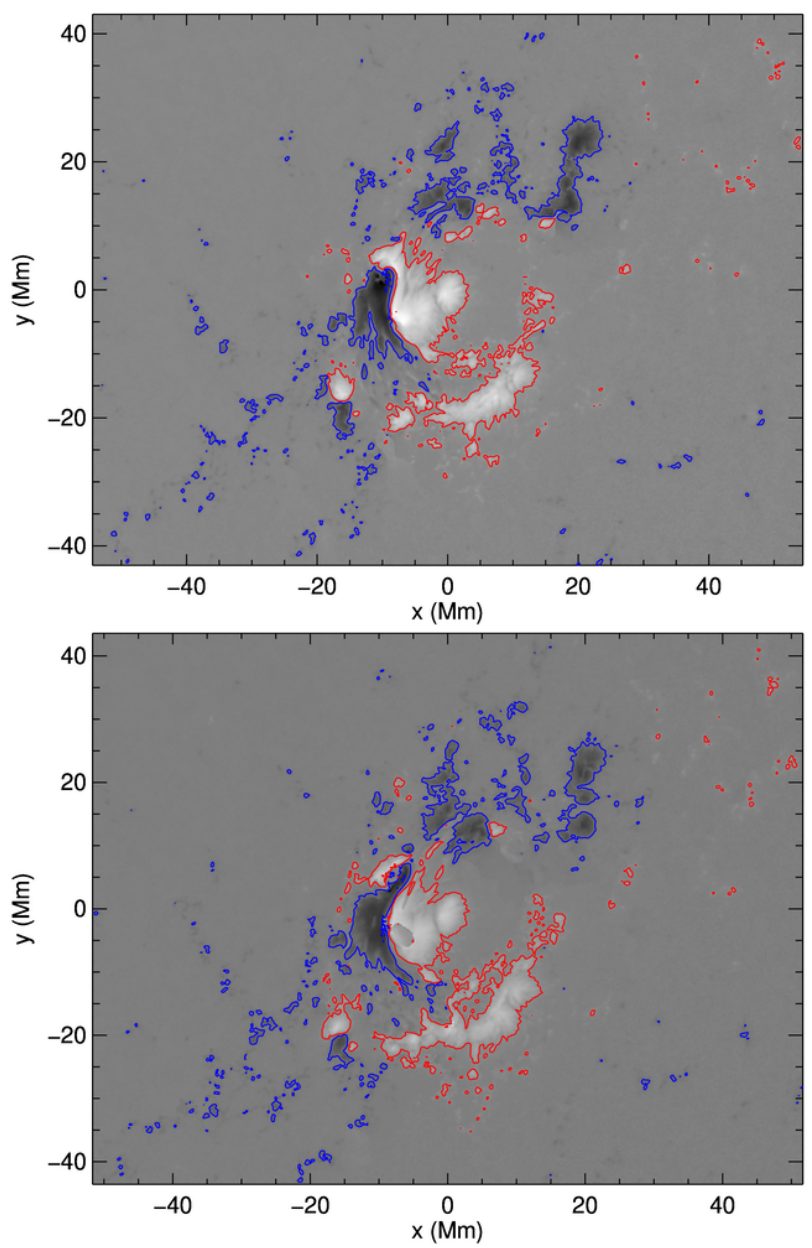

Fig. 2. Photospheric distribution of $B_{z}$ in AR 12673 at the beginning, top plot at 04:12 UT, and the end of our study interval, bottom plot at 13:36 UT. Blue contours correspond to $B_{z}=-500 \mathrm{G}$, and red to $B_{z}=$ $500 \mathrm{G}$.

A possible way to optimize the 3D magnetic field model with respect to its divergence-freeness is to perform different extrapolations and then to retain the one that performs best. We estimated the magnetic field of AR 12673 using two different NLFF methods. The investigation of the various parameters in each method was not exhaustive because our goal was to have a reliable magnetic field for the helicity computations and not to determine it with the highest possible accuracy.

The first extrapolation method that we used was the optimization method of Wiegelmann (2004, hereafter W04). This tries to minimize a functional to fullfil the divergence- and forcefree conditions, given by Eqs. (6), and (7). We note at this point that the assumption that the magnetic field is force free is not always true, especially near the photosphere and during solar flares. For this, the magnetograms were first preprocessed to make them more compatible with the force-free assumption (Wiegelmann et al. 2006) with the standard set of preprocessing parameters $\left[\mu_{1}, \mu_{2}, \mu_{3}, \mu_{4}\right]=\left[1,1,10^{-3}, 10^{-2}\right]$, in the authors' original notation.

The resolution used in the extrapolations was $1^{\prime}$, or $720 \mathrm{~km}$, and the grid size was $320 \times 320 \times 320$ pixels. The computational volume consisted of the volume of interest surrounded by a boundary buffer layer in the lateral and top boundaries with 16 pixels on each side. In the helicity computations only the inner physical part of the fields were kept, after further cutting in height, at roughly two-thirds of the total height. The final 

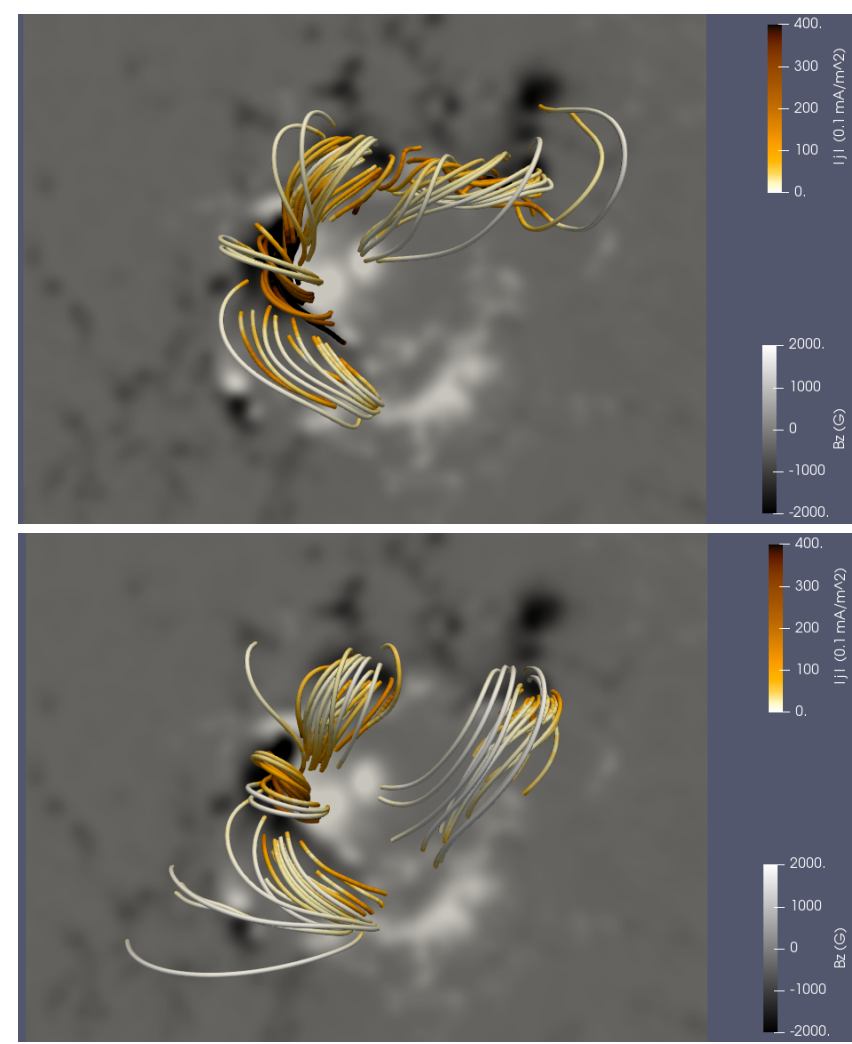

Fig. 3. Morphology of the reconstructed magnetic field of AR 12673 at 08:48 UT on 6 September 2017 with the two versions of the NLFF extrapolation methods (W04 to the top, W12 to the bottom). The same footpoints were used in both cases. Field lines are colored according to the magnitude of the electrical current density.

datacubes thus consisted of $288 \times 288 \times 203$ pixels. The total snapshots were 28 , covering ten hours starting from 6 September 04:00 UT.

The second extrapolation method that we used was the newer version of the same optimization code (Wiegelmann et al. 2012, hereafter W12), which usually performs better. The idea of W12 is to add another term to the functional of W04 to take into account the uncertainties in the measurements of the photospheric magnetic field components. This is done by weighting each pixel separately according to its uncertainty. We used the actual HMI measurement uncertainties for the first time. The empirical weighting function that we employed was

$w=\left\{\begin{array}{ll}0.01+0.99 \exp \left(-\frac{\sigma_{B}}{0.03 B}\right), & \text { pixels with } B \geq 200 \mathrm{G} \\ 0.01, & \text { pixels with } B<200 \mathrm{G}\end{array}\right.$.

Here $B$ denotes the magnetic field strength at a pixel on the photosphere, and $\sigma_{B}$ its uncertainty. We assumed a value of $200 \mathrm{G}$ for the typical noise threshold, and 0.03 for the typical value of $\sigma_{B} / B$. This choice sharply de-emphasizes the contribution of bad measurements in several strong field regions.

All remaining parameters were the same as in the W04 case, except for the size of the buffer, which was 32 pixels in this case. The final datacubes were thus $256 \times 256 \times 203$ pixels. We also used fewer snapshots in this case, 18 instead of 28 , but with the same coverage until the first flare. The morphology of the $3 \mathrm{D}$ magnetic field for the two cases and for the same snapshot is shown in Fig. 3. We note that the two cases exhibit many differences in morphology. The morphology of the magnetic field evolves during the flares, following the evolution of the photospheric magnetic field.
We finally note that we also checked other cases for the extrapolation parameters, such as different $\mu_{3}$ and/or $\mu_{4}$ preprocessing parameters in W04, different binning in W04, and different weighting of the various terms in the functional of W12. None of these cases produced better results, however, and so we present here the two most accurate cases.

\subsection{Solenoidality level estimation}

The resulting magnetic fields were then tested for their quality with respect to how divergence and force free they were. A traditional way to quantify the divergence-freeness of a magnetic field is through the average absolute fractional flux increase, $\left\langle\left|f_{i}\right|\right\rangle$, (Wheatland et al. 2000). This parameter expresses the average of the local non-solenoidalities in the volume of interest; the smaller it is, the more solenoidal the field. The level of divergence-freeness in the W04 case was quite high with a mean value over all the 28 snapshots of $\left\langle\left|f_{i}\right|\right\rangle=(5.9 \pm 0.5) \times 10^{-4}$. The respective values for the W12 set were slightly better, with a mean value over all 18 snapshots of $\left\langle\left|f_{i}\right|\right\rangle=(5.3 \pm 0.6) \times 10^{-4}$.

We also tested the level of force-freeness of the extrapolated fields with an angle, $\theta_{J}$, which expresses the average current-weighted angle between the current and the magnetic field (Wheatland et al. 2000). The mean value we found for the W04 magnetic fields was $\theta_{J}=(16.8 \pm 2.6)^{\circ}$, typical for this method. In the W12 case, the force-freeness level was again slightly better, with an average angle $\theta_{J}=(15.1 \pm 1.5)^{\circ}$.

Another parameter that indicates the divergence-freeness of a magnetic field is the energy ratio $E_{\mathrm{div}} / E$, which was first used by Valori et al. (2016). It expresses the fraction of the total energy that is related to all the (numerical) non-solenoidalities of the magnetic field. To derive the mathematical expression for $E_{\mathrm{div}}$, the magnetic field needs to be decomposed into potential and current-carrying components, $\boldsymbol{B}=\boldsymbol{B}_{\mathrm{p}}+\boldsymbol{B}_{\mathrm{j}}$, and then each component is split into solenoidal and non-solenoidal parts, $\boldsymbol{B}_{\mathrm{p}}=$ $\boldsymbol{B}_{\mathrm{p}, \mathrm{s}}+\boldsymbol{B}_{\mathrm{p}, \mathrm{ns}}$, and $\boldsymbol{B}_{\mathrm{j}}=\boldsymbol{B}_{\mathrm{j}, \mathrm{s}}+\boldsymbol{B}_{\mathrm{j}, \mathrm{ns}}$, following Valori et al. (2013). By defining the energy budget of each component through the relation

$E_{x}=\frac{1}{8 \pi} \int_{\mathrm{V}} \boldsymbol{B}_{x}^{2} \mathrm{~d} V$

we obtained the following decomposition for the total energy of the given magnetic field:

$E=E_{\mathrm{p}, \mathrm{s}}+E_{\mathrm{p}, \mathrm{ns}}+E_{\mathrm{j}, \mathrm{s}}+E_{\mathrm{j}, \mathrm{ns}}+E_{\mathrm{mix}}$,

with $E_{\text {mix }}$ the energy corresponding to all the cross terms. A nonnegative energy associated with all non-solenoidal components of the magnetic field can then be defined by the quantity

$E_{\mathrm{div}}=E_{\mathrm{p}, \mathrm{ns}}+E_{\mathrm{j}, \mathrm{ns}}+\left|E_{\mathrm{mix}}\right|$.

This is an upper limit to the non-solenoidal energy because the absolute value of $E_{\mathrm{mix}}$, the only signed term in the decomposition of Eq. (10), is considered. A perfectly solenoidal magnetic field has $E_{\mathrm{p}, \mathrm{ns}}=E_{\mathrm{j}, \mathrm{ns}}=E_{\mathrm{mix}}=0$, and thus also $E_{\mathrm{div}}=0$; the higher the values of $E_{\mathrm{div}}$, the more non-solenoidal it becomes. Valori et al. (2016) have shown that when $E_{\text {div }} / E$ exceeds $\sim 8-9 \%$, the values of helicity become unreliable.

Because the energy ratio $E_{\text {div }} / E$ is important for helicity computations, we focused more on this quantity. We calculated all components in the energy decomposition following Valori et al. (2013), and we show in Fig. 4 the evolution of $E_{\text {div }} / E$ for the two sets of extrapolated magnetic fields that we used. We also show the condition $E_{\mathrm{div}} / E=0.08$, which, 


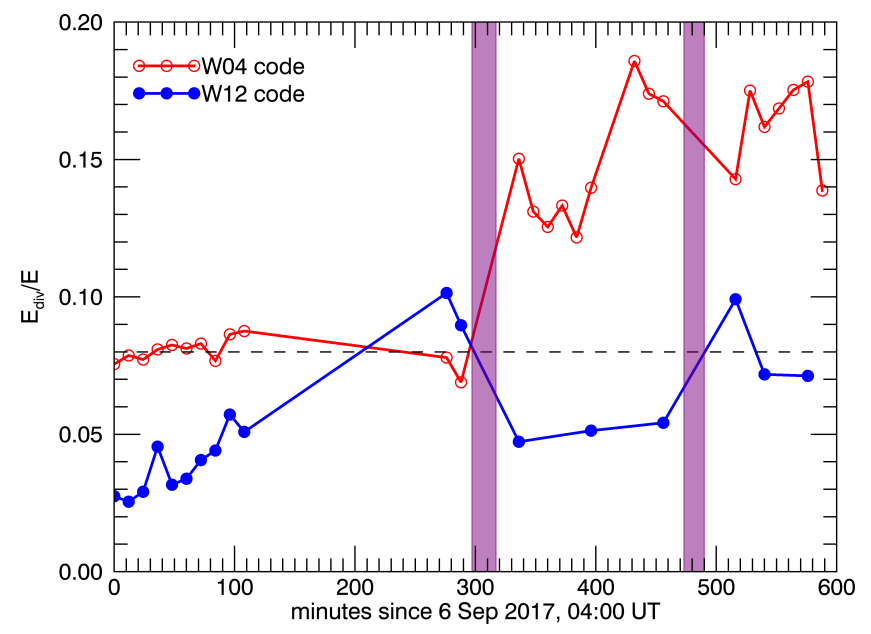

Fig. 4. Evolution of the divergence-related energy normalized to the total field energy in AR 12673 for ten hours around the two X-class flares of 6 September 2017, and for the two sets of extrapolated magnetic fields used. The purple bands denote the time intervals of the X2.2 and the X9.3 flares, with onset times at 08:57 UT and 11:53 UT, respectively.

according to Valori et al. (2016), distinguishes between magnetic fields that lead to reliable values of helicity and those that do not.

We note that the energy ratio in the W04 case performs well until the first $\mathrm{X}$-class flare with values around the limit $E_{\text {div }} / E=0.08$, but all snapshots after the first flare have much higher values. In contrast, most of the snapshots in the W12 case are well below the limit, with the exception of three points: the two immediately before the first flare, and the one after the second flare. Additionally, the values of the energy ratio for the W12 fields are much lower than those of W04, except for the two points before the first flare. Therefore, we kept in the following computations the best-performing snapshots of the W12 method, except from the two snapshots before the first flare, which were replaced by those of W04 that are better, and the snapshot after the second flare where both methods are above the limit and were thus discarded.

\subsection{Magnetic helicity and energy computations}

For each snapshot in the final dataset we computed relative magnetic helicity from its definition, Eq. (1), and also the two gaugeindependent components that it splits into, Eqs. (4), and (5). All the helicities of interest were computed following the method of Moraitis et al. (2014). Briefly, this method first calculates the potential magnetic field that satisfies Eq. (2) so that the relative magnetic helicity given by Eq. (1) is gauge independent. The potential field is obtained from the numerical solution of Laplace's equation with pure Neumann boundary conditions. The two vector potentials are then computed following the recipe of Valori et al. (2012), which makes the clever choice for the gauges, $A_{z}=A_{\mathrm{p}, z}=0$, that results in the straightforward integration of the equations.

We were also interested in the evolution of the various energy budgets of the AR; the total field energy, $E$, the potential energy, $E_{\mathrm{p}}$, including any non-solenoidal contribution it may have, and the free energy, $E_{\mathrm{f}}=E-E_{\mathrm{p}}$. For a perfectly solenoidal magnetic field this free energy coincides with $E_{\mathrm{j}, \mathrm{s}}$ as deduced from Eq. (10), and deviates from it proportionally to the value of $E_{\text {div }}$.
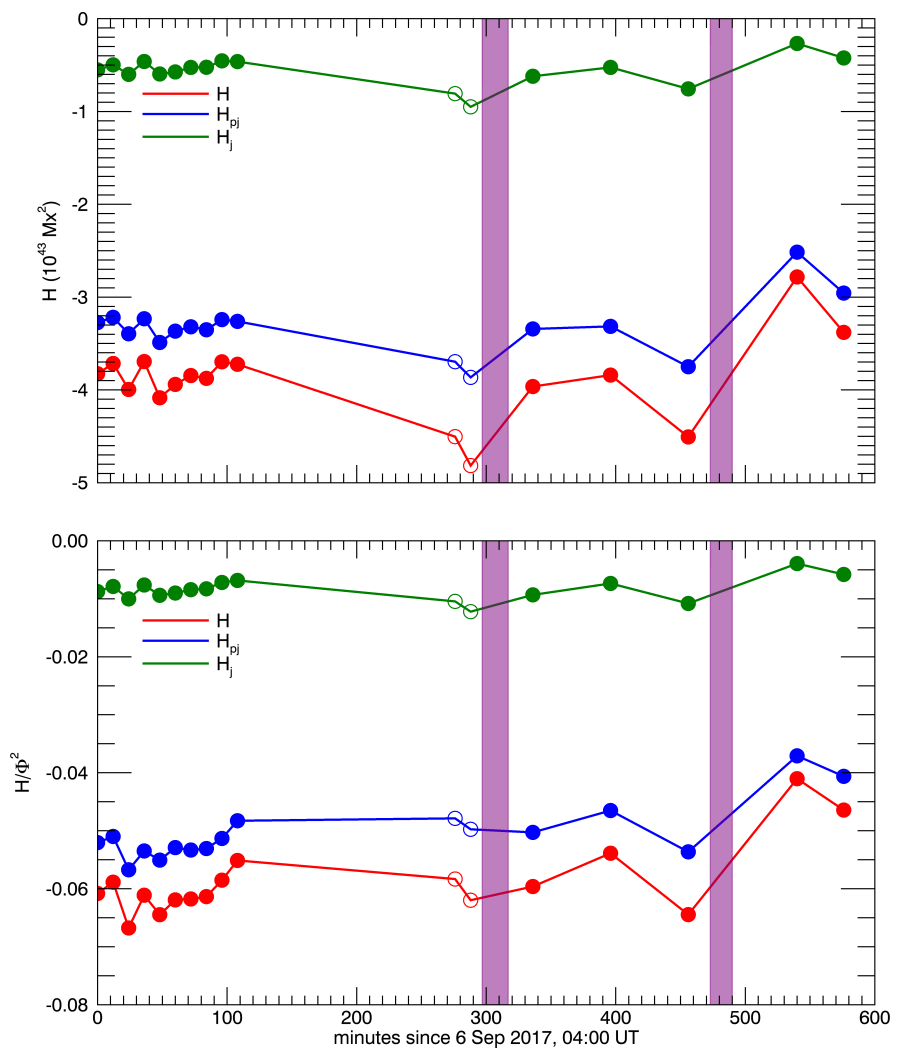

Fig. 5. Evolution of relative magnetic helicity and its two gaugeindependent components in AR 12673 for ten hours around the two X-class flares of 6 September 2017, in physical units (top panel) and normalized to magnetic flux squared (bottom panel). The purple bands denote the time intervals of the X2.2 and the X9.3 flares, with onset times at 08:57 UT and 11:53 UT, respectively. Filled points correspond to the W12 method and open points to the W04 method.

\section{Results}

The evolution of relative magnetic helicity and its two components in AR 12673 is shown in the top panel of Fig. 5. We note that relative magnetic helicity is negative, in agreement with previous studies, and that all helicity budgets are also negative. The absolute values of relative magnetic helicity are very high and reach up to $5 \times 10^{43} \mathrm{Mx}^{2}$, mostly because of the high magnetic flux of the AR. The current-carrying component of helicity is much smaller than the other two helicities, as was also found in other cases (e.g., Moraitis et al. 2014). The evolution patterns are similar for all helicities, increasing before the first flare, relaxing after it, then again increasing before the second flare and relaxing afterwards.

The bottom panel of Fig. 5 shows the helicity budgets normalized to the square of the magnetic flux, $\Phi$, which is calculated from the $B_{z}$ maps of the NLFF fields as $\Phi=\frac{1}{2} \int_{\text {phot }}\left|B_{z}\right| \mathrm{d} S$. The normalized helicity exhibits much more typical values compared to the helicity in physical units, confirming that the high values of helicity are due to the high flux. This plot reveals another feature of the helicity evolution. The normalized helicities have a much smoother evolution than the regular helicities before the first X-class flare, while afterwards, both behave similarly. This could mean that the rise of the helicity budgets before the first flare is related to the increase in flux then, but the later fluctuations are purely flare related.

The evolution of the total field energy, the potential energy, and the free energy is shown in Fig. 6. Again, all budgets attain 


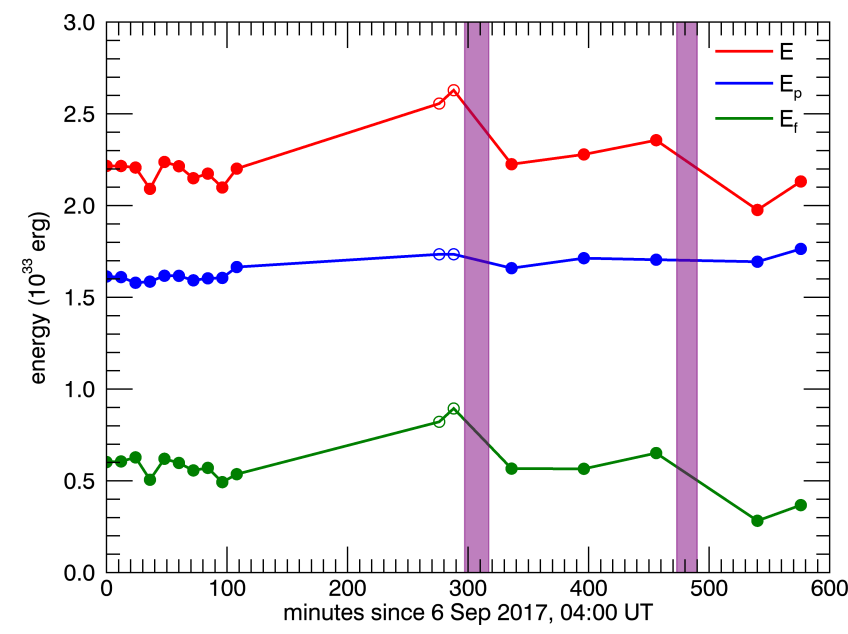

Fig. 6. Evolution of the various energy budgets in AR 12673 for ten hours around the two X-class flares of 6 September 2017. The purple bands denote the time intervals of the X2.2 and the X9.3 flares, with onset times at 08:57 UT and 11:53 UT, respectively. Filled points correspond to the W12 method and open points to the W04 method.

very high values due to the high magnetic flux of the AR. We note that the energy of the potential field changes only slightly during the ten hours of our study, as a consequence of the modest evolution of the normal field on the boundary that is evident in Fig. 2. The change in normal field is less than that in the horizontal field, however, which developed extensive kilogauss bald patches during this period (Sun et al., in prep.). We also note that, although not directly comparable, the evolution pattern of $E_{\mathrm{p}}$ is quite different that the pattern of $H_{\mathrm{pj}}$, even though both quantities involve the potential field. As a result of the small change in potential energy, free energy and total field energy show very similar evolution patterns. Moreover, this pattern resembles the helicity patterns: it increases before the flares and relaxes afterwards.

The main purpose of this work was to examine whether an observed eruptive AR is compatible with the results of Pariat et al. (2017) and Zuccarello et al. (2018), that the helicity ratio $\left|H_{\mathrm{j}}\right| /|H|$ can indicate AR eruptivity. The evolution of this quantity for AR 12673 is shown in Fig. 7. We note that $\left|H_{\mathrm{j}}\right| /|H|$ increases before the two $\mathrm{X}$-class flares and drops after them. The maximum values of the helicity ratio are $\left|H_{\mathrm{j}}\right| /|H| \simeq 0.20$ immediately before the first X-flare, and $\left|H_{\mathrm{j}}\right| /|H| \simeq 0.17$ before the second. Additionally, these two local maxima are the highest values in the helicity ratio time variation. In other words, a threshold in the value of $\left|H_{\mathrm{j}}\right| /|H|$ can be identified from Fig. 7 (red dotted line) above which flares occur. Of course, the exact value of this threshold could depend on the conditions of the specific AR and it should be further examined whether a universal threshold exists. This result seems to be in agreement with the finding of Pariat et al. (2017) that $\left|H_{\mathrm{j}}\right| /|H|$ attains its highest values before eruptions. Moreover, the ratio $\left|H_{\mathrm{j}}\right| /|H|$ rises again at the end of our study interval, possibly indicating the subsequent activity of AR 12673.

Figure 7 also displays the evolution of the ratio of free energy to total energy, $E_{\mathrm{f}} / E$, in AR 12673 . This parameter has also been examined by Pariat et al. (2017) and Zuccarello et al. (2018), and it was found to be less indicative of eruptivity than $\left|H_{\mathrm{j}}\right| /|H|$. The energy ratio evolution pattern that we find exhibits some similarities to the helicity ratio pattern, which could be a result of the relation between free energy and relative magnetic helicity magnitude (Tziotziou et al. 2012, 2014). The local maximum of the energy ratio before the second flare is much weaker than the corresponding local maximum of the helicity ratio, however.

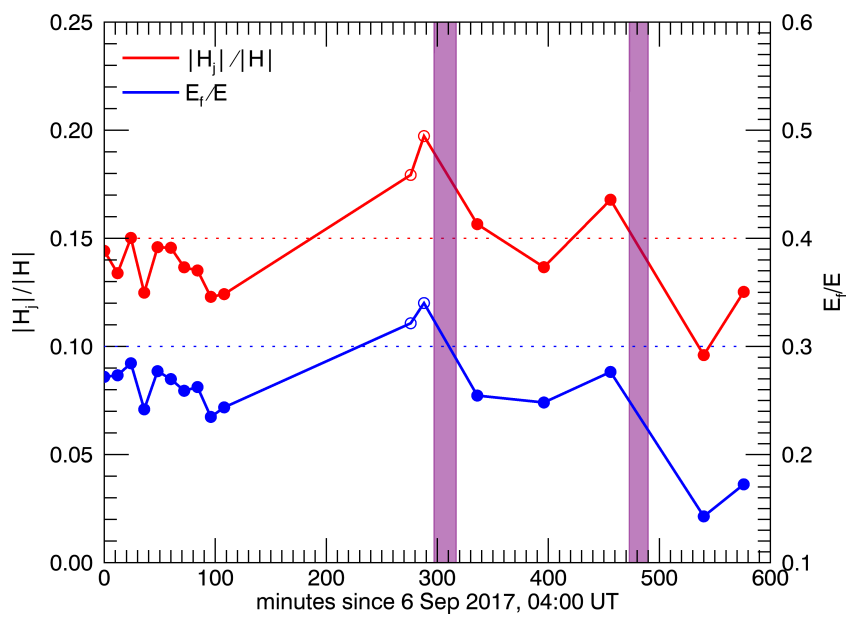

Fig. 7. Evolution of the ratios $\left|H_{\mathrm{j}}\right| /|H|$ and $E_{\mathrm{f}} / E$ in AR 12673 for ten hours around the two X-class flares of 6 September 2017. The purple bands denote the time intervals of the X2.2 and the X9.3 flares, with onset times at 08:57 UT and 11:53 UT, respectively. Filled points correspond to the W12 method and open points to the W04 method.

We note in Fig. 7 that free energy, from the peak of $\sim 34 \%$ of the total energy before the first flare, drops to $\sim 25 \%$ between the two flares, and finally to $\sim 15 \%$ after the second. Differently than the helicity ratio, therefore, the energy ratio cannot be used to set a threshold above which flares occur.

\section{Discussion}

We reported the detailed study of the helicity and energy properties of AR 12673 at the time around its two consecutive X-class flares of 6 September 2017. All involved quantities were accurately computed for the whole period of intense activity. This was done for the first time in such detail. The computations followed a recent finite volume method, and the careful modelling of the 3D magnetic field of the AR.

In the latter, the coronal magnetic field was extrapolated with two different versions of an optimization-based NLFF method. In the newer version of the method, an observationally derived empirical weighting that employs the HMI uncertainty estimate was used for the first time on such methods. This version is the most accurate, as deduced by a parameter that distinguishes extrapolations that lead to reliable helicity values from those that do not, but not in all the cases examined. The older version performed better in two snapshots immediately before the first X-class flare. The best-performing NLFF fields from the two methods were compiled into the final dataset we used.

In a very recent work (Thalmann et al. 2019), the authors suggested that the threshold in the parameter $E_{\text {div }} / E$ should be lower that the threshold we used, $5 \%$ instead of $8 \%$, for an NLFF field to be used in helicity studies. We expect to see differences of about $4-5 \%$ to the derived helicities with such an NLFF field, as follows from Valori et al. (2016), and our results are therefore probably not affected by this change.

The modelling of the magnetic field enabled us to follow the evolution of the relative magnetic helicity and its two components, the non-potential and the volume-threading component, in AR 12673. The negative sign of all helicity components confirmed the findings of other authors. The values of relative helicity that we estimated are similar in magnitude to those reported by Vemareddy (2019); they reach up to $-5 \times 10^{43} \mathrm{Mx}^{2}$. They were three orders of magnitude higher than those of Yan et al. (2018), 
however, although both cited works used the flux-integration method to compute the helicity. Compared to Liu et al. (2018), our helicity values are again much higher, but these authors approximated helicity only with its twist, and so the difference is reasonable. The change in helicity during the first flare that we found was also much smaller than theirs.

Additionally, we examined the evolution of the various energy budgets in AR 12673. The evolution pattern of the free energy that we derived seems to agree with the results of Mitra et al. (2018), although a direct comparison is not possible because the authors only provide the ratio $E_{\mathrm{f}} / E_{\mathrm{p}}$. The field energy that we derive, which is as high as $2.5 \times 10^{33} \mathrm{erg}$, also compares well with that of Vemareddy (2019), which was computed as the sum of the accumulated energy in the system.

The focus of this work was examining the behaviour of the helicity ratio $\left|H_{\mathrm{j}}\right| /|H|$ during the two flares. This first observational determination of the evolution of this ratio in an AR seems to confirm the findings of Pariat et al. (2017): the helicity ratio increases before major flares, and it relaxes afterwards. Moreover, the much smaller current-carrying part of helicity, that is, the fact that $\left|H_{\mathrm{j}}\right| /|H|<1$, is in agreement with previous results (Moraitis et al. 2014). The examination of the energy ratio $E_{\mathrm{f}} / E$ is also compatible with the results of Pariat et al. (2017) and Zuccarello et al. (2018). This ratio is also related to AR eruptivity, but to a lesser extent than $\left|H_{\mathrm{j}}\right| /|H|$, and without the capability of providing a threshold above which flares occur, unlike the helicity ratio.

In order to establish these results, the evolution of the helicity ratio needs to be examined in a large number of ARs, with different characteristics regarding their evolutionary stage and/or their eruptivity, and with the highest possible cadence. A step in this direction is made with the work of Thalmann et al. (in prep.). We provide the first observational support here that the helicity ratio $\left|H_{\mathrm{j}}\right| /|H|$ is related to solar eruptivity.

Acknowledgements. E. Pariat, L. Linan, and K. Moraitis acknowledge the support of the French Agence Nationale pour la Recherche through the HELISOL project, contract $\mathrm{n}^{\circ}$ ANR-15-CE31-0001. X. Sun is partially supported by NSF award 1848250 . This work was supported by the Programme National PNST of CNRS/INSU that is co-funded by CNES and CEA.

\section{References}

Augusto, C. R. A., Navia, C. E., de Oliveira, M. N., et al. 2019, PASP, 131, 024401

Berger, M. A. 1999, Plasma Phys. Control. Fusion, 41, B167

Berger, M. A., \& Field, G. B. 1984, J. Fluid. Mech., 147, 133

Chertok, I. M., Belov, A. V., \& Abunin, A. A. 2018, Space Weather, 16, 1549

Finn, J., \& Antonsen, T. 1985, Plasma Phys. Control. Fusion, 9, 111

Green, L. M., Török, T., Vršnak, B., Manchester, W., \& Veronig, A. 2018, Space Sci. Rev., 214, 46

Hou, Y. J., Zhang, J., Li, T., Yang, S. H., \& Li, X. H. 2018, A\&A, 619, A100

Inoue, S., Shiota, D., Bamba, Y., \& Park, S.-H. 2018, ApJ, 867, 83

James, A. W., Valori, G., Green, L. M., et al. 2018, ApJ, 855, L16

Linan, L., Pariat, É., Moraitis, K., Valori, G., \& Leake, J. 2018, ApJ, 865, 52

Liu, L., Cheng, X., Wang, Y., et al. 2018, ApJ, 867, L5

Mitra, P. K., Joshi, B., Prasad, A., Veronig, A. M., \& Bhattacharyya, R. 2018, ApJ, 869, 69

Moraitis, K., Tziotziou, K., Georgoulis, M. K., \& Archontis, V. 2014, Sol. Phys., 289, 4453

Morosan, D. E., Carley, E. P., Hayes, L. A., et al. 2019, Nat. Astron., 3, 452

Nindos, A., \& Andrews, M. D. 2004, ApJ, 616, L175

Pariat, É., Valori, G., Démoulin, P., \& Dalmasse, K. 2015, A\&A, 580, A128

Pariat, É., Leake, J. E., Valori, G., et al. 2017, A\&A, 601, A125

Pesnell, W. D., Thompson, B. J., \& Chamberlin, P. C. 2012, Sol. Phys., 275, 3

Rust, D. M. 1994, Geophys. Res. Lett., 21, 241

Scherrer, P. H., Schou, J., Bush, R. I., et al. 2012, Sol. Phys., 275, 207

Sun, X., \& Norton, A. A. 2017, Res. Notes Am. Astron. Soc., 1, 24

Taylor, J. B. 1974, Phys. Rev. Lett., 33, 1139

Thalmann, J. K., Linan, L., Pariat, E., \& Valori, G. 2019, ApJ, 880, L6

Tziotziou, K., Georgoulis, M. K., \& Raouafi, N.-E. 2012, ApJ, 759, L4

Tziotziou, K., Moraitis, K., Georgoulis, M. K., \& Archontis, V. 2014, A\&A, 570, L1

Valori, G., Démoulin, P., \& Pariat, É. 2012, Sol. Phys., 278, 347

Valori, G., Démoulin, P., Pariat, É., \& Masson, S. 2013, A\&A, 553, A38

Valori, G., Pariat, É., Anfinogentov, S., et al. 2016, Space Sci. Rev., 201, 147

Vemareddy, P. 2019, ApJ, 872, 182

Verma, M. 2018, A\&A, 612, A101

Veronig, A. M., Podladchikova, T., Dissauer, K., et al. 2018, ApJ, 868, 107

Wang, H., Yurchyshyn, V., Liu, C., et al. 2018, Res. Notes Am. Astron. Soc., 2, 8

Wheatland, M. S., Sturrock, P. A., \& Roumeliotis, G. 2000, ApJ, 540, 1150

Wiegelmann, T. 2004, Sol. Phys., 219, 87

Wiegelmann, T., Inhester, B., \& Sakurai, T. 2006, Sol. Phys., 233, 215

Wiegelmann, T., Thalmann, J. K., Inhester, B., et al. 2012, Sol. Phys., 281, 37

Yan, X. L., Wang, J. C., Pan, G. M., et al. 2018, ApJ, 856, 79

Yang, S., Zhang, J., Zhu, X., \& Song, Q. 2017, ApJ, 849, L21

Zuccarello, F. P., Pariat, E., Valori, G., \& Linan, L. 2018, ApJ, 863, 41 